\title{
Herbal Products and Conventional Drugs - an Uneasy Alliance
} Glynn $J^{1}$, Bhikha $R^{2}$

\begin{abstract}
This review examines the interaction between herbal remedies and conventional (orthodox) drugs. There has been a substantial increase in the consumption of herbal remedies in recent times for various reasons. They are often combined with one another, and are often used simultaneously with conventional drugs that are taken for a wide range of disorders, both acute and chronic. The probability of a real and substantial interaction between the therapeutic agents originating from different medical paradigms is likewise expanding. Whilst most of the herb-drug interactions are undoubtedly minor, benign, and harmless, there are reports of more serious interactions. Possible reasons for specific herb-drug interactions are examined, particularly from the pharmacokinetic and pharmacodynamics perspectives.
\end{abstract}

Keywords: herbals; pharmaceuticals; interaction; pharmacokinetics; pharmacodynamics.

International Journal of Human and Health Sciences Vol. 02 No. 04 October'18 Page : 193-198 DOI: http://dx.doi.org/10.31344/ijhhs.v2i4.55

\section{Introduction}

There has been a major resurgence of interest in herbalism in the last 30 or 40 years. ${ }^{1}$ This interest may be directed towards herbalism itself or to it as a popular component of complementary medical practice. $^{2}$ This trend has been given impetus by real misgivings about the negative aspects of numerous conventional drugs. There is growing concern about the short term downside to drugs, especially the newer ones, regarding the impact of adverse drug reactions, and suspicions about the real extent to which they occur. ${ }^{3}$ It reflects the urge towards a more natural lifestyle, and a new-found respect for natural or holistic healing. Herbal medicine deals with the underlying disharmony which manifests during the onset of a chronic or recurring disease. ${ }^{4}$ This 'whole picture' approach differs from that of conventional medicine, which is concerned primarily with alleviating the patient's symptoms and normalizing signs such as laboratory parameters. Unlike with conventional medicine, the administration of herbal products, alone or as part of a broader complementary or traditional medical strategy, is a good example of holism in action. Herbs are increasingly being used these days as therapeutic agents, or combined with minerals and vitamins in health enhancing supplements, or in invigorating tonics and teas. Although the benefits of herbal medicine are vast, it is essential to understand the basics of herbal medicine and to accept that some herbal medicines can have negative impacts on health, especially if conventional drugs are being consumed at the same time. One of these relates to herb-drug interaction.

\section{Background}

Herbal medicine, like many natural or traditional therapies, has been around for many hundreds of years. Virtually every society or culture has adopted it in one form or another. Even today, in spite of the massive inroads achieved by conventional medicine and synthetic drug usage, around $80 \%$ of the world's population uses it to a greater or lesser extent. ${ }^{5,6}$ It may be stand-alone therapy, especially for the minor complaints affecting us all, or it may be part of a more comprehensive complementary healing system, where it is combined with traditional systems such as Tibb or Ayurveda for chronic and recurring disorders. ${ }^{7}$ Until well into the latter

1. John Glynn, IbnSina Institute of Tibb, 1137 Anvil Road, Robertville, Roodepoort, Johannesburg, South Africa (Researcher)

2. Rashid Bhikha, IbnSina Institute of Tibb, 1137 Anvil Road, Robertville, Roodepoort, Johannesburg, South Africa, (Chairperson)

Correspondence to: Rashid Bhikha, IbnSina Institute of Tibb, 1137 Anvil Road, Robertville, Roodepoort, Johannesburg, South Africa, Email: rashidb@tibb.co.za; Phone: 0119917323. 
part of the $20^{\text {th }}$ century, complementary medicine in general and herbal medicine in particular took a 'back seat', especially in the developed world, and was perceived as the poor cousin to conventional, orthodox or western medicine. The increasingly sophisticated but destructive practice of many alternative practitioners - "quacks" - who systematically sullied the herbalists' reputation, encouraged, understandably, a fierce backlash against complementary medicine which accelerated its decline in usage. There were also numerous other obstacles in the way of the widespread use of herbal products. ${ }^{1}$ They included reliability and variation of pharmacological action, lack of credibility, possible toxicity, and doubts about the authenticity of the product. Even so, a number of herbs have now established themselves in the therapeutic mainstream as both effective and safe for defined clinical disorders. ${ }^{5}$ This has led to many people combining herbal medicines with conventional drugs in a healing practice known as Integrative Medicine, resulting in increased frequency of herb-drug interactions. ${ }^{8}$ Most of these are minor, often barely noticeable. However, some interactions can not only affect clinical response, but also lead to troubling adverse reactions or side effects. This review examines the nature of these interactions, and how they manifest.

\section{Herbal medicines}

A herb, as described in the Concise English Dictionary, is any non-woody, seed-bearing plant which dies down to the ground after flowering, whose leaves, seeds, flowers, or other parts can be used to improve health, combat disease, for food and its flavoring, and improve environmental quality with scents.

Herbal medicines are finished, labelled medicines containing pharmacologically active parts of a plant or plants, either in crude form or physically modified during processing. They are usually administered orally or topically to deal with chronic or acute clinical disorders and are available as fresh or dried plants, or as tablets, capsules, powders, teas, or extracts. ${ }^{9}$

They are based on certain parts of the medicinal plant - flower, leaf, stem, bark, or root. However, they are not single substances extracted by chemical techniques from the plant and subsequently purified or even structurally altered. The use of herbal remedy is ideally combined with lifestyle advice - changes to food consumption, rest, exercise, for example. This enhances the potency of the herbal medicine by supporting and boosting the patient's innate self-healing processes and so helping restore normal harmony and rhythm. ${ }^{10}$

Herbal remedies do not subscribe to the "quick fix' philosophy of conventional medicine. By adopting herbal medicine integrated with lifestyle modification, the chronic malady is not only more likely to be ameliorated, but the chances of recurrence greatly reduced. ${ }^{10}$

\section{Overview of conventional drugs}

By conventional drugs we mean either (a) pharmacological single entities which have been derived by chemical synthesis - the so-called newto-nature drugs; or (b) single chemical derivatives of naturally occurring pharmacologically active substances detected in, and isolated from, members of the plant, fungus, bacterial, or animal world. ${ }^{11,12}$ The first category includes laboratory originated drugs such as the ACE inhibitors, beta blockers, proton pump inhibitors, and nonsteroidal anti-inflammatories. The second category includes morphine, theophylline, colchicine, and other drugs derived from plant alkaloids; immune suppressants such as cyclosporine from soil fungus, most antibiotics, and human hormones and cytokines, such as insulin and interferon. They may be used singly, or, more often, they may be administered combined with other conventional drugs. This is done either to enhance the desired clinical effect, or to ameliorate the negative side effects of the principal drug.

Generally speaking, the prime objective of conventional drug therapy is to alleviate the patient's troubling symptoms. ${ }^{13}$ The relief of pain, depression, digestive disorders, movement problems, for example, is paramount. The secondary objective is to restore the presenting signs to normal. These days this usually refers to one or more of the battery of laboratory parameters detected in the patient's body fluids, soft tissues and organs, and skeleton. There is, however, no real or urgent imperative to deal with the underlying causes of the disorder, whether lifestyle, environmental, or behavioral in origin. This contrasts markedly with the objectives of herbal therapy. This accepts the need for the relief of the patient's symptoms, but equally accepts the need to rectify the underlying disharmony in the patient which has encouraged the development of the disorder in the first place. This may be humoral in nature, or biochemical, temperamental, or due to a dysfunctional self-healing apparatus.

\section{Herb - drug interactions}

As there is a significant and increasing number 
of people taking herbal remedies simultaneously with conventional drugs, there is a distinct and real possibility of herb-drug interaction, analogous to the drug-drug interaction that is now well established and documented.

It is generally accepted that drugs can interact with food. MAO- inhibitors are potentiated by some cheeses; antibiotic absorption is inhibited by milk; and warfarin action is diminished by green vegetables. ${ }^{14}$ The original indication that a herbal agent could interact significantly with the action of a conventional drug was the observation, more than 20 years ago, that the consumption of grapefruit juice could markedly reduce the metabolism of the immunosuppressant cyclosporine in patients following transplantation..$^{15}$ We now know that the metabolic enzymes (comprising the cytochrome P450 system) of the patient's liver were being inhibited, so higher levels of the active drug were being maintained for a longer period of time. As a result, a lower dose of the (very expensive) drug could be employed without compromising clinical efficacy or outcome. The cost-effectiveness implications are substantial.

If grapefruit has such an important effect - why not herbs? For most herb-drug interactions the actual mechanisms involved are not fully understood. What we do know is that the drug does not combine with a herb component to form a toxic intermediary. ${ }^{8}$ In most cases changes to the absorption of a drug, or its metabolism within the body, is most likely the basis for interaction. We know that several herbs can induce or inhibit enzymes, resident in the liver or intestinal tract walls, which are responsible for drug metabolism. These enzymes, which are mainly of the cytochrome P450 type, are made less effective, so the level of unmetabolised drug rises within the systemic blood circulation, and clearance of the unchanged drug and its metabolites is affected. ${ }^{16}$ Numerous clinical trials, case studies, and anecdotal case reports have emerged which attest to the unequivocal interaction of herbs with drugs. ${ }^{17,18}$ This is only to be expected; if a herb actually triggers a pharmacological response, then it could conceivably interfere, to a greater or lesser extent, with a conventional drug's activity. This has important ramifications for drug therapy: many people are taking not one, but several, potent drugs. The potential for herb-drug interaction is therefore immense when the number of herbal products coming into popular use is considered. St John's wort is arguably the 'poster child' of herb- drug interactions. ${ }^{19,20}$ It has been linked to a whole range of drug interactions: certain anxiolytics, lipid lowering agents, immune-suppressants, antiretroviral, anti-diabetic agents, oral contraceptives, and others. ${ }^{21}$ Over the last few years a number of popular herbs - garlic, ginkgo, and ginseng, for example - have been shown to interact with specific drugs. Some of the interactions are of minor significance, whereas others do carry important, serious cautions. The drugs that seem to be most involved in interactions are blood thinners such as warfarin ${ }^{22}$, antibiotics, and certain antidepressants ${ }^{21}$.

The interaction of herbs with drugs appears to be highly specific. That is, an interaction will only become significant or clinically important if a critical aspect of the drug's pharmacological action is interfered with. For example, if a herb affects a drug's absorption rate, it will only be a cause for concern if it is important for the drug to be absorbed quickly (as with analgesics), or totally (as with antibiotics). If the absorption rate is not a critical factor (as with antidepressants or anxiolytics) then the interaction is likely to be of minor importance.

Garlic is known to decrease absorption of the antiretroviral agent saquinovir, and is implicated in the onset of hypoglycemia in diabetic patients taking chlopropamide. In its turn, ginkgo increases clotting time in patients taking aspirin or warfarin, and is implicated in increasing blood pressure in hypertensive patients taking a thiazide diuretic. Ginseng, another very popular herb, also interferes with warfarin activity, digoxin levels, and certain antidepressants. ${ }^{23}$

The implications of the herb-drug interaction are determined by the degree of interaction, the extent of the interaction in people using the two, and the seriousness of the adverse effect. For instance, hypericum is now linked to breakthrough bleeding and unwanted pregnancies in women taking certain oral contraceptives. In this situation, the use of the herbal product should only be taken for good reason, and with alternative contraception adopted. This constraint would also apply to Parkinson's disease patients being treated with L-dopa, where kava increases the 'off' periods of treatment benefit. ${ }^{24}$

Not all herb-drug interactions are unwelcome or deleterious. ${ }^{25,26}$. Piperine (an active ingredient of peppers) increases the bio-availability of the anti-epileptic drug phenytoin, the beta blocker propanolol, and the bronchodilator theophylline. 
In patients with pulmonary tuberculosis, ginseng increases rifampicin blood levels. Regarding drug toxicity, huangqinis effective in ameliorating the digestive problems associated with irinotecan in patients with cancer.

\section{Modes of action of herbs vs drugs}

Identifying the pharmaceutical mechanisms of herbal products activity poses numerous challenges not faced by those studying conventional drugs. For example, herbal products contain many active substances which can act in combination or synergistically, whereas conventional drugs are generally studied in isolation as single agents. Another difference is that a single conventional drug may only have one major direct action on a particular receptor site. Indeed, the more specific for one type of receptor is the preferred outcome of research for new pharmaceutical agents. Conversely, herbal products probably have several different pharmacological actions, and the one that predominates depends on the dosage employed, the part of plant selected, and the presence of other actives. Moreover, the herbal product may contain a particular active agent, but it may not be present in sufficient quantity to elicit a pharmacological action. Yet another difference is that pharmacological synergy may operate with a multi-component herbal product - that is, the net pharmacological response will be different when compared to one active agent alone, as the dominant mechanism may be potentiated by a separate mechanism.

If a disorder is complex in origin, as with many chronic or recurring ailments, a simple approach such as drug treatment is unlikely to be more than marginally effective. Such disorders do not usually arise from a single biochemical, systemic, or organic defect, because the body's inner healing power is quite capable of dealing with this. Clinical disorders are the result of a complex disturbance of homeostasis, involving numerous cellular and organic networks. Most disorders are the body's natural response to several factors, some small, but others sometimes large, which work together to overcome the body's inner healing capacity. There may be trigger factors; environmental causes, genetic susceptibility and interplay, dietary factors, emotional considerations, lifestyle issues, all conspiring together.

In order to modify and negate the disease process, effective therapy depends on addressing more than one form of disharmony. Herbal remedies usually offer a multi-target approach, in contrast to conventional drugs, which generally affect the workings of one receptor site, or the action of one enzyme. What herbs do is mimic the body's natural intrinsic healing processes. This dilemma has long been recognized by conventional healthcare practitioners, and an attempt to resolve it by resorting to drug combinations, or poly-pharmacy. Cocktails of potent drugs are now almost standard in treating cancer, tuberculosis, HIV \&Aids, heart disease, and others.

Mechanisms of drug-herb interaction

The mechanisms by which a herbal product interferes with a drug, or vice versa, are not fully understood in many cases. There are, however, a number of possible activities which explain the interaction; these are divided into pharmacokinetic and pharmacodynamics interactions.

\section{Pharmacokinetic interaction}

Inhibition of absorption. The herb or drug acts on the other active agent to reduce or minimize its absorption. A number of agents have been identified:

- Pectins, resins, tannins may bind to certain antibiotics, preventing unimpeded absorption of the drug.

- Garlic (Allium sativum) interacts with several drugs. It also reduces plasma concentrations of chlorzoxazone.

- Ginkgo decreases the plasma concentrations of many drugs.

[In practice this interaction can best be avoided by separate timing of drug and herb administration]

Enhancement of absorption. This is usually the result of increased gastric emptying or shorter gut transit time.

- Laxatives can increase these processes.

- Certain saponins can increase gastric emptying, so enhancing the rate of gastric emptying.

[In practice this interaction can best be avoided by reducing laxative usage, or selecting alternate times, or by dividing doses between morning and evening]

Drug metabolism increased. The herbal product promotes an increase in metabolic enzymes in the liver, so boosting the breakdown or elimination of drugs.

- St John's wort reduces the plasma concentrations and increases clearance of a wide range of drugs.

- Echinacea affects the metabolic clearance of caffeine and certain anti-anxiety drugs.

- Ginkgo decreases the plasma concentrations of many drugs. 
- Grapefruit juice decreases metabolism of cyclosporine and other drugs, due in induction of liver metabolic CYT P450 enzyme.

[In practice this interaction can best be avoided by monitoring plasma drug levels, and adjusting dosage appropriately]

Binding of drug by a herb component. Some antibiotics are chemically bound to certain botanical substances. This prevents or inhibits absorption through the intestinal wall, as the drugherb complex is either too big to absorb, or too poorly soluble in the intestinal fluid.

[In practice this interaction can best be avoided by separating the timing of dosing of the drug and the herbal product]

\section{Pharmacodynamics interaction}

Electrolyte depletion. Laxatives and diuretic drugs can reduce sodium and potassium levels in the body, and so lead to adverse cardiac effects.

[In practice this interaction can best be avoided by separating the timing of dosing of the drug and the herbal product].

The pharmacological effect is intensified. Certain herbs have specific pharmacological effects. If a drug is being co-administered, an additive or perhaps synergistic effect can arise.

- Several herbal products and certain drugs may have the same effect on blood clotting mechanisms.

- Hypoglycemic drugs and herbal products used to treat diabetes may act together.

- Herbs which contain the alkaloids caffeine and ephedrine, or similar, may increase nerve stimulant drug action.

- St John's wort may enhance the nervous system effects of certain antidepressant drugs.

[If both drug and herb are being used for the same therapeutic objective, a decision has to be made preferring one active agent based on cost, ADRs, long term effects].

\section{Summary}

There are thousands of herbs with a wide range of different uses, many of which are used in conventional medicine as well as for natural remedies. Herbal medicine is one of the oldest forms of medicine used for the treatment of physical as well as psychological maladies and in the search for optimum health. It has played a major part in medicine for millennia. As the use of herbal medicine is on the increase, the possibility of interaction with conventional drugs is real, and can pose a threat to successful treatment. Herbal medicines possess significant pharmacological activity, so can interact markedly with many conventional drugs if these are being taken simultaneously. Many conventional drugs are used in the general population, especially in the elderly, and these people often resort to herbal medicine for improved success in treating chronic disorders in particular. It is therefore important to assess whether a particular herbal remedy interferes with the expected effect of a conventional drug (and vice-versa), and if this can lead to unexpected and unwanted adverse effects. 


\section{$\underline{\text { References }}$}

1. Ekor M. The growing use of herbal medicines: issues relating to adverse reactions and challenges in monitoring safety. Front Pharmacol. 2013;4:177.

2. Rosenfeld I. Guide to alternative medicine. Random House, London, UK, 1996.

3. Pirmohamed M, James S, Meakin S et al. Adverse drug reactions as cause of admission to hospital: prospective analysis of 18820 patients. BMJ. 2004;329:15.

4. Van der Merwe A. Herbal Remedies. Tafelberg, Cape Town, South Africa, 2002 .

5. Wachtel-Galor S, Benzie IFF. Herbal Medicine: Biomolecular and Clinical Aspects. (2nd edition). CRC Press, France, 2011.

6. Si-Yuan P, Litscher G, Si-Hua G, et al. Historical Perspective of Traditional Indigenous Medical Practices: The Current Renaissance and Conservation of Herbal Resources. Evidence-based Compl\&Altern. Med. 2014.

7. Bhikha RAH, al Haq MAH, Glynn JP. Integrative medicine, chronic disease and Tibb. World Review of Science, Technology and Sustainable Development. 2008;(5) 2

8. $\mathrm{Hu} \mathrm{Z}$, Yang, $\mathrm{X}$ et al . Herb-drug interactions: A literature review. Drugs. 2005;65(9):1239-82.

9. Ody P. The Herb Society's Complete Medicinal Herbal. Human \& Rousseau, Cape Town. 1993.

10. Roy R. Integrative medicine to tackle the problem of chronic diseases. J. Ayurveda Integr. Med. 2010;1(1 );

11. Grifo F, Rosenthal J. (Eds) Biodiversity and Human Health. Island Press, Washington DC .

12. Goodman and Gilman's Manual of Pharmacology and Therapeutics (2nd Ed.) McGraw-Hill, New York.2014

13. Boissel JP. Note on the necessary definition of therapeutic objectives for optimal prescription. Therapie. 1996;51(3):287-289.

14. McCabe BJ. Prevention of food-drug interactions with special emphasis on older adults. Curr. Opin. Clin. Nutr. \& Metabol. Care. 2004;7(1); 21-26.
15. Hollander AA et al. The effect of grapefruit juice on cyclosporine and prednisone metabolism in transplant patients. Clin Pharmacol Ther. 1995;57(3):318-324.

16. Choi YH, Chin YW, Kim YG. Herb-drug interactions focus on metabolic enzymes and transporters. Arch Pharm. Res. 2011;34(11): 1843-1863.

17. Cupp MJ. Herbal remedies: adverse effects and drug interactions. AmerFam Physician. 1999;59(5);12391244.

18. Fugh-Berman A. Herb-drug interactions. Lancet. 2000;8:355(9198);134-138.

19. Ranjat L. Review: Hyperfine: a potent anti-depressant natural drug. Intern. J. Pharm. and Pharmaceut. Sci. 2013;5(3):

20. Zhou S, Chan Eli, Pan S-Q et al . Pharmacokinetic Interactions of Drugs with St John's Wort. Ann. Rev Pharmacol Toxicol 2004;39:361-398.

21. Pozadski P, Watson L, Ernst E. Herb-drug interactions: an overview of systematic reviews. Brit J. Clin. Pharmacol. 2013;76(3); 603-618.

22. Biekang G, Khang Z, Zuo Z. Updates on the Clinical Evidenced Herb-Warfarin Interactions. Evidencebased Complem. Alt. Med. 2014; 957362

23. Vaes LP, Chyka PA. Interactions of warfarin with garlic, ginger, ginkgo or ginseng: nature of the evidence. Ann Pharmaco therap. 200;34;1478-1482.

24. Rodda HC, Kumar, Samala $S$ et al . An Insight into Herb - Drug Interactions. Intern. J. Pharma. Scien. and Nanotech. 2010;2(4);

25. Nargis N, Al Mahmood Abu K, Afrin SF, Sayeed MH, Hassa Md Z. Unani preparation 'Sharbat Misali' is useful as an alternate medicine to safely treat anemia: A pilot study. Bangladesh Journal of Medical Science, 2018;17(1):144-148.

26. Sørensen JM. Herb-Drug, Food-Drug, NutrientDrug, and Drug-Drug Interactions: Mechanisms Involved and Their Medical Implications J. Altern. And Complem. Med. 2004;8(3):293-308. 